\title{
Volume and heat of solution of hydrogen in rare earths from proton screening charges
}

\author{
J.M. Marchetti ${ }^{\mathrm{a}}$, S. Segui ${ }^{\mathrm{b}}$, J.L. Gervasoni ${ }^{\mathrm{b}, \mathrm{c}, *, 1}$, A. Juan ${ }^{\mathrm{a}, 1}$, J.P. Abriata ${ }^{\mathrm{b}}$ \\ ${ }^{a}$ Departamento de Fisica, Universidad Nacional del Sur, 8000 Bahia Blanca, Argentina \\ ${ }^{\mathrm{b}}$ Centro Atómico Bariloche, Comisión Nacional de Energía Atómica, 8400 S.C. Bariloche, Argentina \\ ${ }^{\mathrm{c}}$ Instituto Balseiro, Universidad Nacional de Cuyo, 8400 S.C. Bariloche, Argentina
}

Received 16 December 2005; received in revised form 6 March 2006; accepted 7 March 2006

\begin{abstract}
In this work we determine some fundamental microscopic and macroscopic properties of rare earth-hydrogen (RE-H) systems. The behaviour of the electronic density variation of RE-H systems is obtained, using a program based on the density functional formalism. This information allows us to calculate the volume of solution of hydrogen in rare earths, as well as their heat of solution, and to compare with experimental results.
\end{abstract}

(C) 2006 Elsevier Ltd. All rights reserved.

Keywords: A. Metals; C. Ab initio calculations; D. Defects

\section{Introduction}

The technological interest for rare earths has been stimulated by different processes which use individual rare earths as metals and as salts. One of these applications is the use of mixed rare earths (mischmetal) for petroleum cracking.

Another application of great interest is the interaction of rare earths with hydrogen. This is particularly true in the case of the study of rare-earth hydrides for the storage of hydrogen as a source of energy. One example of this is the increasing development of $A B_{5}$ type alloys, where $A$ is a rare earth or a rare-earth mix, and $B$ is a $3 d$ metal, such as nickel. The study of these compounds comes from the need of substituting petroleum by cleaner and cheaper fuel for internal combustion engines. These technological developments are also associated to rare earths extraction and purification costs [1].

\footnotetext{
*Corresponding author. Centro Atómico Bariloche, Comisión Nacional de Energía Atómica, 8400 S.C. Bariloche, Argentina. Tel.: + 542944445118 ; fax: +542944445190 .

E-mail address: gervason@cab.cnea.gov.ar (J.L. Gervasoni).

${ }^{1}$ Also member of the Consejo Nacional de Investigaciones Científicas y Técnicas.
}

The study of hydrided pure rare earths constitutes the fundamental tool in order to understand the basics of the hydriding process, and a good starting point to extrapolate results to alloys. In the present work, we propose a first approximation to the study of properties such as the volume of solution and the heat of solution of the RE-H systems using a simple model to describe the electronic density.

\section{Theoretical model}

To characterize the properties of hydrogen solution in rare earths we assume that the electron density can be described by an effective jellium model [2,3]. In this model, which has proved to be adequate for some transition metals, the valence electrons are regarded as a free electron gas of density $n_{0}$, with a background of uniform and positive charge of the same density, to keep the neutrality of the system. This is reasonable when the main contribution to the electron density comes from $s$-electrons or from closed-shell configurations, with spherical symmetry. Nevertheless, it has proved to be useful for other electronic configurations as long as the properties studied are derived from averaged microscopic quantities. 
In a free electron gas, the density $n_{0}$ is given by $n_{0}=3 /\left(4 \pi r_{s}^{3}\right)$, where $r_{s}$ is the radius of a sphere corresponding to one electron in the gas. The value of $r_{s}$ is one of the fundamental parameters of the jellium model and it is necessary to assess it with great accuracy. Unfortunately, the available data for rare earths are scarce, and we have to determine it in an indirect way. In the case of metals, Serebrinsky et al. [4] proposed a parameterization to obtain the value of $r_{s}$ from the bulk modulus $B$, which is a well measured physical quantity for practically all the elements through the periodic table. In this work, we adopt this modelization, restricting it to rare earths to obtain new values of the fitting parameters.

The parameterization given in [4] relies on the definition of $B$ in terms of the energy $\varepsilon$ per particle of the electrons in the gas. This energy can be written as the sum of the kinetic $\varepsilon_{k i n}$, exchange $\varepsilon_{x}$ and correlation $\varepsilon_{c}$ energies, and each one as a function of $r_{s}$. The bulk modulus $B$ is obtained as the simple sum of the corresponding contributions $B_{k i n}, B_{x}$ and $B_{c}$. The parameterization given in [4] is

$B\left(r_{s}\right)= \begin{cases}B_{k i n}+B_{x}+B_{c}, & r_{s} \leqslant 1, \\ B_{k i n}+\frac{1}{r_{s}}\left(B_{x}+B_{c}\right), & r_{s}>1,\end{cases}$

where

$B_{k i n}\left(r_{s}\right)=\frac{1}{2 \pi} \frac{1}{\alpha^{2} r_{s}^{5}}, \quad B_{x}=-\frac{1}{2 \pi^{2}} \frac{1}{\alpha r_{s}^{4}}, \quad B_{c}=B_{c}\left(r_{s}\right)$

and $\alpha=(4 / 9 \pi)^{1 / 3}$. The correlation energy $\varepsilon_{c}$ is always negative and its functional form depends on the range of $r_{s}$ considered. At metallic densities, it goes from 0.1 to $0.5 \varepsilon_{x}$, but in the limit of high densities it is proportional to $\ln r_{s}$. An analogous relation can be assumed for $B_{c}$ and $B_{x}$.

In the case of rare earths elements and their precursors (Sc, Y, and La), the available experimental data for $B[6,7]$ show a variation from $\approx 13.5 \mathrm{GPa}$ (for Ytterbium) to $\approx$ $45 \mathrm{GPa}$ (for Scandium). Taking into account that for metals these values correspond to $r_{s}$ ranging from $\approx$ 3.1 a.u. to $\approx 2.7$ a.u., ${ }^{2}$ we follow the functional dependence previously obtained for metals and propose a parameterization given by

$B\left(r_{s}\right)=B_{k i n}+\frac{b_{0}}{r_{s}^{5}}$.

The values of $r_{s}$ used for the fitting are those obtained from the material density (see e.g. [5]). The experimental data here used for the bulk modulus $B$ are those given by Gschneidner [6]. Although this database may seem too old, the results obtained with the more recent (but incomplete) database given by Hachiya and Ito [7] differ in less than $0.5 \%$. The value of the fitting parameter $b_{0}$ is -0.0307881 a.u.. In Fig. 1 the function obtained is shown, as well as the contribution of the kinetic term alone $\left(B_{k i n}\right)$,

\footnotetext{
${ }^{2} \mathrm{We}$ use atomic units for the $r_{s}$ values since the figures are more convenient. The length atomic unit is the Bohr radius $a_{0}=5.292 \times 10^{-11} \mathrm{~m}$.
}

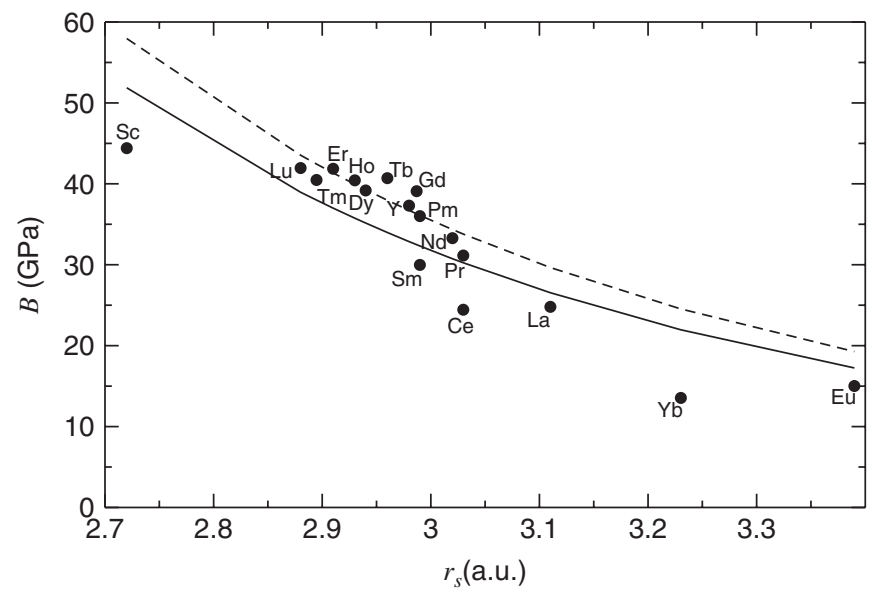

Fig. 1. Bulk modulus vs. $r_{s}$. Continuous line: this work (Eq. (2)); dashed line: kinetic contribution $B_{k i n}$; circles: experimental data for $B$ [6].

and they are compared with the experimental data. The values for $r_{s}$ show little dispersion and the relative differences are in general less than 0.04 (except for $\mathrm{Yb}$, with 0.09).

\section{Results}

The semiempirical parameterization of $B$ as a function of $r_{s}$ proposed in the previous section provides a simple definition for $r_{s}$. This parameter can be used in a variety of calculations in which the electronic density is perturbed in the interstitial region. This is the case for a hydrogen atom interstitially dissolved in a material host. This impurity produces an expansion of the lattice represented by the volume of solution $V_{H}$. The calculation of this quantity is quite complicated and very difficult to perform in a systematic way through the periodic table.

With the calculated data for $r_{s}$ we use the jellium model [2] to obtain the density of charge $Q$ induced by a proton embedded in a rare earth host. The induced charge density allows us to obtain the volume of solution of hydrogen $V_{H}$, as explained in Section 3.1. Moreover, we obtain the variation of the total energy of the system, from which we obtain the heat of solution $\Delta H$ in Section 3.2.

It is worth pointing out that the calculations presented here do not take into account the magnetic properties of the rare earth considered. This is permissible in a first approximation, since the studied quantities are not strongly dependent on the magnetic behaviour of the material host.

\subsection{Application to the calculation of $V_{H}$}

The change of energy $\delta E$ associated with an inhomogeneus (but isotropic) deformation introduced by one hydrogen atom in the material determines the volume of solution through the expression [8] 
$v_{H}=\frac{V_{H}}{\Omega}=\frac{1}{\Omega} \frac{\delta \Omega}{\delta P} \sum_{i} \frac{\delta \mathbf{R}_{i}}{\delta \Omega} \cdot \frac{\delta E}{\delta \mathbf{R}_{i}}=-\frac{1}{B} \sum_{i} \frac{1}{3} \frac{\mathbf{R}_{i}}{\Omega} \cdot \frac{\delta E}{\delta \mathbf{R}_{i}}$,

where $\Omega$ is the volume of the unit cell, $B$ is the bulk modulus and $\delta \mathbf{R}_{i}$ is the deformation at the positions $\left\{\mathbf{R}_{i}\right\}$ of the ions in the host. To first order in $\left\{\delta \mathbf{R}_{i}\right\}$, and using the Hellman-Feynmann theorem [2,3], $\delta E$ is given by the electrostatic interaction between the ionic cores of the host and the impurity of charge $Z_{0}$ at $\mathbf{r}=0$

$\delta E=\sum_{i} Z_{i}\left(\int \frac{\Delta n(\mathbf{r})-Z_{0} \delta(\mathbf{r})}{\left|\mathbf{r}-\mathbf{R}_{i}\right|^{3}}\left(\mathbf{R}_{i}-\mathbf{r}\right) \mathrm{d}^{3} r\right) \delta \mathbf{R}_{i}$.

Here $Z_{i}$ is the charge of the ion located at $\mathbf{R}_{i}$ and $\Delta n(r)$ is the charge density induced by the impurity, which depends on the effective parameter $r_{s}$.

In the case studied here, with a hydrogen atom embedded in a jellium, these expressions are simplified and the value of $V_{H}$ can be calculated as

$V_{H}=\frac{1}{3 B} 4 \pi n_{0} \lim _{R \rightarrow \infty} \int_{0}^{R}[1-Q(r)] r \mathrm{~d} r$,

where $Q(r)$ is the integrated screening charge density.

Nørskov and Lang [9] and Stott and Zaremba [10] obtained a parameterization for the energy of an atom embedded in an inhomogeneus system, using the theory of the effective medium. In the present work, we used a computer code based on this model to obtain $Q(r)$ in order to evaluate $V_{H}$ for rare earths.

In Fig. 2, calculated values of $V_{H}$ are plotted as a function of the atomic number $Z$. In general, they show an approximately constant behaviour, with a mean value around $V_{H} \approx 4.5 \AA^{3}$. It is worth pointing out that the numerical program used to calculate the electronic density and the charge distribution does not show a clear convergence in some particular cases $(\mathrm{Eu}$ and $\mathrm{Yb})$. The calculation of $V_{H}$ showed to be very sensitive to the value of the integral in Eq. (5). The discontinuities appearing in the plot $V_{H}$ vs. $Z$ for these elements correspond to discontinuities of $r_{s}$ as a function of $Z$ : while most of the rare-earth elements present a value of $r_{s}$ approximately

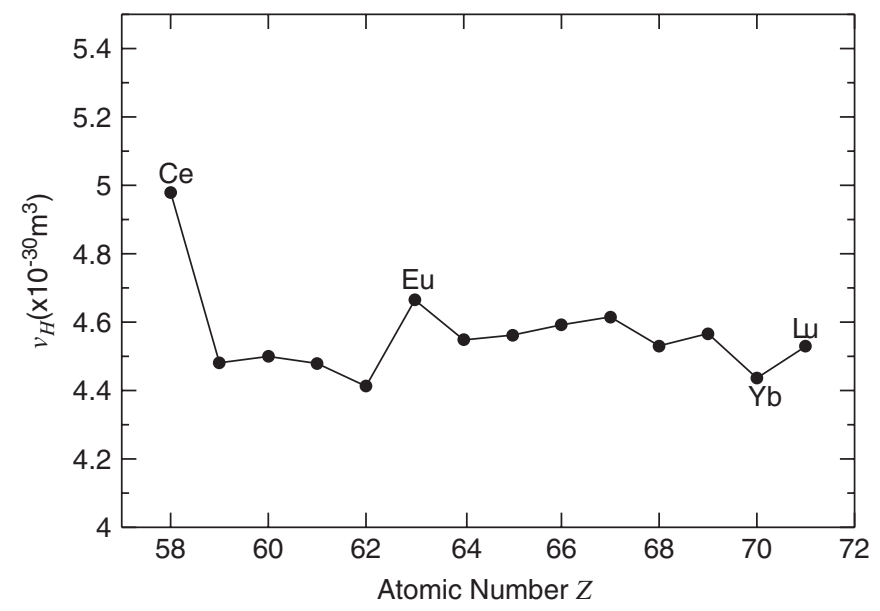

Fig. 2. Calculated values of $V_{H}$ as a function of the atomic number $Z$. constant $(\approx 2.9$ a.u. $)$, the values for $\mathrm{Eu}$ and $\mathrm{Yb}$ are above $3.5 \AA$. Correspondingly, the values of the bulk modulus for these elements are considerably lower than the mean of the remaining elements.

Besides, the volume of solution for Cerium seems to be too high when comparing with the remaining elements of the group. This behaviour is not surprising if we consider the itinerant character of the $4 f$-electron, which is not taken into account by the model.

The experimental values for $V_{H}$ corresponding to rare earths are scarce and show considerable dispersion. In Table 1, a comparison of calculated values with experimental data is shown, for the rare earth precursors Sc, Y and $\mathrm{La}[1,11,12]$, and for $\mathrm{Er}, \mathrm{Tm}$ and $\mathrm{Lu}[13,14]$. As can be seen, the prediction is reasonable although it may seem to overestimate the value of $V_{H}$. It is worth pointing out that the data taken from Ref. [11] correspond to dihydrides, which may not be well described by our infinite-dilution model.

Besides, comparing experimental values obtained by different authors for Yttrium, we observe that the value obtained by Griessen and Feenstra is $1.1 \AA^{3}$ while Khatamian's value is $3.00 \AA^{3}$. The explanation for this discrepancy is the following: Khatamian's data for $V_{H}$ are obtained from a measure of samples of composition $\mathrm{YD}_{0.176}$, whereas Griessen and Feenstra took for $V_{H}$ the difference in molar volume between $\mathrm{YH}_{2}$ and $\mathrm{Y}$. The calculation is then as follows: atomic volume of pure $\mathrm{Y}$ : $33.034 \AA^{3}$; atomic volume of $\mathrm{YH}_{2}: 35.345 \AA^{3}$; the difference in volume divided by 2 gives $V_{H}=1.16 \AA^{3}$.

For metallic elements, the volume of solution $V_{H}$ shows a smooth behaviour as a function of $r_{s}$ and can be parameterized in terms of the linearized Thomas-Fermi model [4], considerably simplifying the calculations. In order to compare this result with our calculations for rare earths, in Fig. 3 the calculated values of $V_{H}$ are plotted as a function of $r_{s}$. As can be seen, they are quite well approximated by the parameterization proposed by Serebrinsky et al.

Besides the particular cases indicated above, the jellium model implemented here gives reasonable predictions for

Table 1

Calculated and experimental values of $V_{H}$, given in $\AA^{3}$

\begin{tabular}{lllll}
\hline Sample & $r_{s}($ calc $)$ & $V_{H}($ calc $)$ & $V_{H}(\exp )$ & Reference \\
\hline Sc & 2.79 & 4.49 & 2.36 & {$[1]$} \\
& & & 1.1 & {$[11]$} \\
$\mathrm{Y}$ & 2.90 & 4.35 & 1.1 & {$[11]$} \\
& & & 3.00 & {$[12]$} \\
$\mathrm{La}$ & 3.14 & 5.10 & 4.25 & {$[1]$} \\
$\mathrm{Er}$ & 2.85 & 4.53 & 3.97 & {$[13]$} \\
& & & 3.06 & {$[14]$} \\
$\mathrm{Tm}$ & 3.55 & 4.57 & 3.61 & {$[13,14]$} \\
$\mathrm{Lu}$ & 2.83 & 4.52 & 3.25 & {$[13]$} \\
& & & 2.72 & {$[14]$} \\
\hline
\end{tabular}

Note that experimental values given in Ref. [11] correspond to dihydrides. 


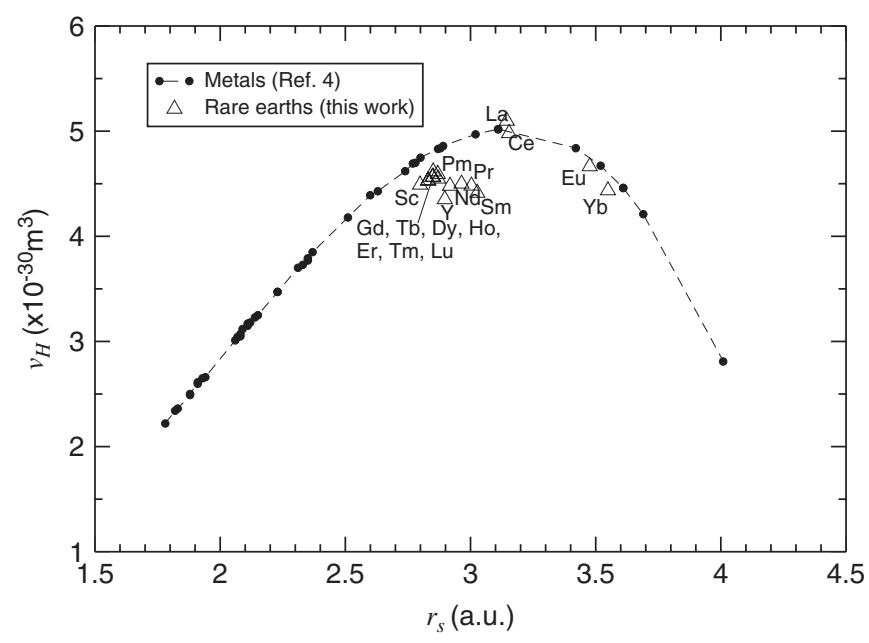

Fig. 3. Calculated values of $V_{H}$ as a function of the electronic radius $r_{S}$.

the volume of solution for the rare earth group. Despite its simplicity, it seems to be an adequate tool to make a first approximation to the problem studied here.

\subsection{Heat of solution $\Delta H$}

Another quantity of interest in the study of the system RE-H is the heat of solution $\Delta H$, that is, the variation of the enthalpy of the system when an atom of hydrogen is embedded in the host. Griessen and Driessen [15] proposed an empirical linear relation for obtaining the heat of formation of metal hydrides from a characteristic energy $\Delta E$ of the electronic band structure of the metal host,

$\Delta H=\frac{n_{s}}{2}(a \Delta E+b)$,

where $n_{s}$ is the number of electrons per atom in the lowest $s$-like conduction band of the host. The values of the fitting parameters given by these authors are $a=$ $29.62 \mathrm{~kJ} \mathrm{eV}^{-1} \mathrm{~mol} \mathrm{H}^{-1}$ and $b=-135 \mathrm{~kJ} \mathrm{~mol} \mathrm{H}^{-1}$. An improvement of this model has been developed by Griessen [16] for transition metals, taking into account the width of the d-band and the local environment of the hydrogen atom in the metal host.

The heat of formation of hydrides (which can be symbolized by $\mathrm{MH}_{x}$, with $x \geqslant 1$ ) may differ markedly from the heat of solution of the systems studied here, where the hydrogen is infinitely diluted $(x \rightarrow 0)$. In analogy with the work of Griessen and Driessen, we propose here a linear relation between the heat of solution and the variation of the total energy of the system $\left(\Delta E_{T}\right)$ produced when embedding a hydrogen atom in the host, as given by the jellium model. This variation of the energy is assumed to be the sum of the changes in the kinetic energy of the noninteractive system of the electron gas, the exchange and correlation energies and the classical electrostatic energy.

Although the experimental data available for $\Delta H$ corresponding to rare earths are scarce, their behaviour

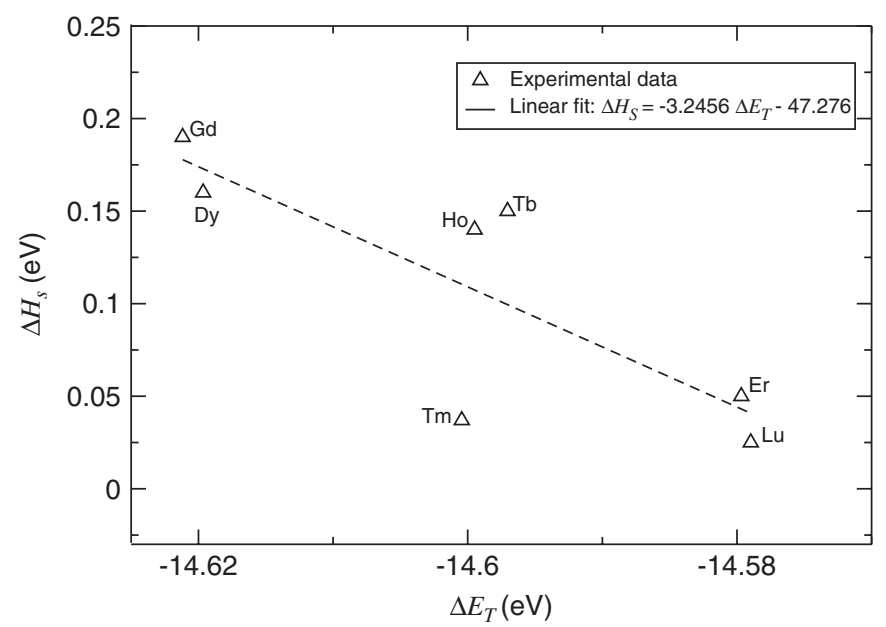

Fig. 4. Linear fit of experimental values [17] for $\Delta H$ as a function of $\Delta E_{T}$.

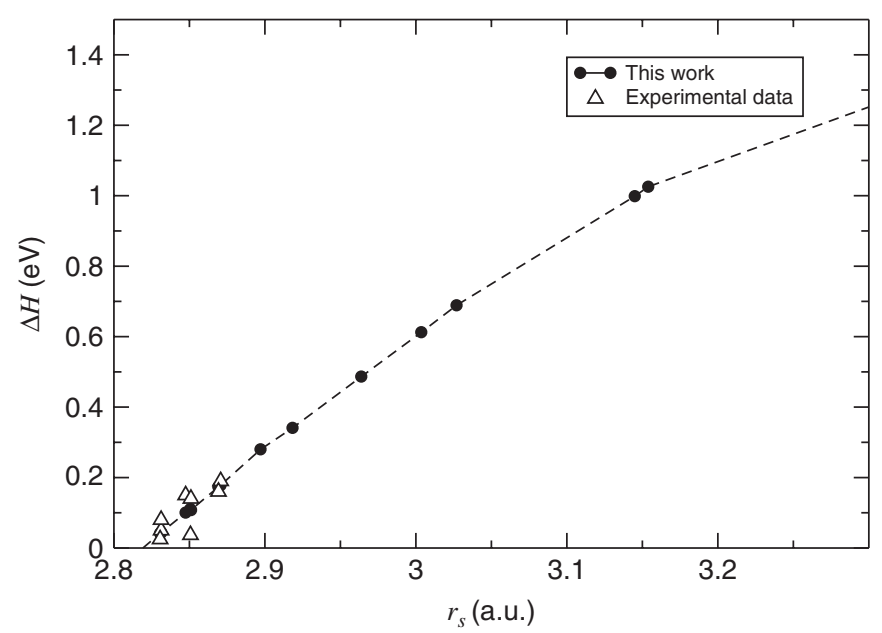

Fig. 5. Heat of solution $\Delta H$ as a function of the electronic radius $r_{s}$. Experimental values compiled by Vajda [17].

when plotted against our calculated values for $\Delta E_{T}$ (see Fig. 4) allows us to propose

$\Delta H=\tilde{a} \Delta E_{T}+\tilde{b}$

with $\tilde{a}=-2.40$ and $\tilde{b}=-34.92$ (with both quantities, $\Delta E_{T}$ and $\Delta H$ given in $\mathrm{eV}$ ).

In Fig. 5, calculated values for $\Delta H$ corresponding to lanthanides are plotted as a function of the electron radius $r_{s}$. Also displayed in Fig. 5 are the experimental values compiled by Vajda [17]. Although the experimental data span a reduced range of $r_{s}$, the predicted behaviour seems to reproduce the correct trend. Nevertheless, it would be desirable to count with newer data for comparison.

\section{Conclusions}

We have presented a simple and general method for obtaining the volume and the heat of solution of hydrogen in rare earths. Although both quantities are rather difficult 
to measure in these elements, they are very important because they determine several properties of the hydrogenrare earth systems.

Since our calculations are valid for infinite solid solution at zero temperature and pressure, we should compare only with highly diluted $\mathrm{RH}_{x}$ systems, with $x$ tending towards zero. Nevertheless, experimental values correspond to finite $x$, and $T$ and $P$ different from zero, and we have to take these features into account when we compare both results. We also include dihydride values to have a feeling of the validity of our approximation in such cases. From the theoretical point of view it is still necessary to develop a more realistic description to explain why, in the specific case of Yttrium, hydrogen has a large partial volume (about 3) at low $x$ and a value three times smaller for dihydride. Furthermore, in the $\mathrm{CaF}_{2}$ phase $\left(\mathrm{YH}_{2}\right)$ there is a small contraction, with increasing $x$ (see Fig. 9 of Vajda's review paper).

However, for the studied quantities $\left(V_{H}\right.$ and $\left.\Delta H\right)$, the global agreement with existing experimental data is very reasonable. The discrepancy between any particular element corresponds to corrections due to effects not considered here, as for example those associated to the strain energy or to the hydrogen-core (or semi-core) interactions.

In summary, our method is simple, useful and can be advantageously used as long as neither sufficiently accurate first principle calculations nor experimental data are available.

\section{Acknowledgments}

We would like to thank Dr. P. Vajda (École Politechnique, France) and R. Griessen (Vrije Universitaet, The Netherlands) for their useful comments.
Financial support from the Agencia Nacional de Promoción Científica y Tecnológica (ANPCYT, PICT 15047 and PICT 1209857) is gratefully acknowledged. SS also wishes to thank a fellowship from ANPCYT (PICT 126328).

\section{References}

[1] Y. Fukai, Springer Series in Materials Science 21: The MetalHydrogen System - Basic Bulk Properties, Springer, Berlin, 1993.

[2] P. Hohenberg, W. Kohn, Phys. Rev. 136 (1964) B864.

[3] W. Kohn, L.J. Sham, Phys. Rev. 140 (1965) A1133.

[4] S.A. Serebrinsky, J.L. Gervasoni, J.P. Abriata, V.H. Ponce, J. Mater. Sci. 33 (1998) 167.

[5] N.W. Ashcroft, D. Mermin, Solid State Physics, Saunders College Publishing, New York, 1976.

[6] K.A. Gschneidner, in: F. Seitz, D. Turnbull (Eds.), Solid State Physics. Advances in Research and Applications, Academic Press, New York and London, 1964, p. 275.

[7] K. Hachiya, Y. Ito, J. Phys. Condens. Matter 11 (1999) 6543.

[8] G. Leibfried, N. Breuer, Point defects in metals I-Introduction to the theory, Springer Tracts in Modern Physics, vol. 81, Springer, Berlin, 1993.

[9] J.K. Nørskov, N.D. Lang, Phys. Rev. B 21 (1980) 2131.

[10] M.J. Stott, E. Zaremba, Phys. Rev. B 22 (1980) 1564.

[11] R. Griessen, R. Feenstra, J. Phys. F 15 (1985) 1013.

[12] D. Khatamian, C. Stassis, B.J. Beaudry, Phys. Rev. B 23 (1981) 614.

[13] B.J. Beaudry, F.H. Spedding, Metall. Trans. 6 (1975) 419.

[14] J.E. Bonnet, J. Less 49 (1976) 451.

[15] R. Griessen, A. Driessen, Phys. Rev. B 30 (1984) 4372.

[16] R. Griessen, Phys. Rev. B 38 (1988) 3690.

[17] P. Vajda, in: K.A. Gschneidner, L. Eyring (Eds.), Handbook on the Physics and Chemistry of Rare Earths, vol. 20, North-Holland, Amsterdam, 1995, p. 207. 